\title{
Praktische Aspekte der pharmakologischen antiarrhythmischen Therapie im Kindes-und Jugendalter
}

Bei Kindern und Jugendlichen mit strukturell normalem Herzen sind die Mechanismen, die zu Arrhythmien führen, häufig dieselben wie bei Erwachsenen. Einige der Arrhythmien sind eher mit jungem Lebensalter assoziiert und man sieht sie selten im Erwachsenenalter.

Davon abzugrenzen sind Arrhythmien bei Kindern mit angeborenen Herzfehlern. Hier entstehen Arrhythmien entweder in Assoziation mit dem vorliegenden strukturellen Defekt oder als Ergebnis chirurgischer Interventionen und damit verbundener Narben. Chronischer hämodynamischer Stress kann bei diesen Kindern ebenso das Risiko für Arrhythmien erhöhen.

Die Art der medikamentösen Akut- und auch Dauertherapie/Dauerprophylaxe im Kindes- und Jugendalter lehnt sich dabei größtenteils an die der Erwachsenentherapie an. Dies hängt mit der mangelnden Datenlage zur Behandlung von Arrhythmien im Kindesalter zusammen. Evidenz zur Wirksamkeit und Sicherheit der Therapien, erhoben in pädiatrischen Studien, beziehen sich hauptsächlich auf Beobach- tungsstudien, die fast alle retrospektiver Natur sind. Einige wenige kontrollierte randomisierte Doppelblindstudien finden sich vor allem im Bereich der Therapie supraventrikulärer Tachykardien. In der pädiatrischen Population findet sich die höchste Inzidenz von supraventrikulären Tachykardien bei Neugeborenen und Säuglingen.

Als allgemeine Regel benötigt der Beginn einer antiarrhythmischen Dauertherapie die Stellung einer klaren Diagnose mittels EKG-Dokumentation der zu behandelnden Arrhythmie. Nach Durchführung einer Risiko-Nutzen-Abwägung wird die Therapie eingeleitet und über einen Zeitraum durchgeführt, bis sich die Krankheit der Erfahrung nach selbst limitiert, was bei Neugeborenen und Säuglingen mit strukturell gesundem Herzen häufig der Fall ist, oder bis eine kurative Therapie in Form einer Katheter-Ablation indiziert ist und durchgeführt werden kann.

Basierend auf dem Consenus Statement der EHRA/AEPC von 2013 und anhand der Analyse der bis heute publizierten Da- ten wird Prof. N. Sreeram die aktuellen Handlungsempfehlungen und praktischen Aspekte der pharmakologischen antiarrhythmischen Therapie im Kindes- und Jugendalter in seinem Vortrag präsentieren.

\section{Interessenkonflikte}

Der Autor gibt an, dass kein Interessenkonflikt besteht.

\section{Autor}

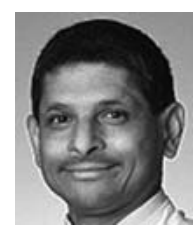

Narayanswami Sreeram Klinik und Poliklinik für Kinderkardiologie, Universitätsklinikum Köln

\section{Korrespondenzadresse}

Prof. Dr. med. Narayanswami Sreeram Klinik und Poliklinik für Kinderkardiologie Universitätsklinikum Köln Joseph-Stelzmann-Straße 9 50924 Köln

narayanswami.sreeram@uk-koeln.de 